\title{
Entropy production and Onsager reciprocal relations describing the relaxation to equilibrium in stochastic thermodynamics
}

\author{
Stefano Giordano* \\ Univ. Lille, CNRS, Centrale Lille, Univ. Polytechnique Hauts-de-France, \\ UMR 8520 - IEMN - Institut d'Électronique de Microélectronique et de Nanotechnologie, LIA LICS/LEMAC, F-59000 Lille, France
}

(Dated: April 2, 2021)

\begin{abstract}
We study the relation between stochastic thermodynamics and non-equilibrium thermodynamics by evaluating the entropy production and the relation between fluxes and forces in a harmonic system with $N$ particles in contact with $N$ different reservoirs. We suppose that the system is in a non-equilibrium stationary state in a first phase and we study the relaxation to equilibrium in a second phase. During this relaxation, we can identify the linear relation between fluxes and forces satisfying the Onsager reciprocity and we obtain a nonlinear expression for the entropy production. Only when forces and fluxes are small, the entropic production turns into a quadratic form in the forces, as predicted by the Onsager theory.
\end{abstract}

\section{INTRODUCTION}

At the beginning of the 19th century, the out-of-equilibrium statistical mechanics received a major boost from the experimental observations of the Brownian motion [1, 2], and the membrane hydrodiffusion [3]. The first theories to explain these phenomena were developed by Einstein [4, 5], Smoluchowski [6], and Langevin [7], which laid the foundations for the use of stochastic processes in physics. Importantly, these approaches provided a definitive test of the atomic hypothesis [8]. Starting from this historical milestone, the Langevin stochastic equation was widely used and its properties were studied by introducing the probability density of the variables involved [9-13], thus developing the modern FokkerPlanck theory [14-17]. More recently, Sekimoto introduced the concepts of heat and work for a given stochastic trajectory [18, 19], creating a conceptual link between thermodynamics and stochastic evolution of a system. Similarly, the concepts of entropy (total entropy, entropy flow and entropy production) were associated with Langevin trajectories, complementing the aforementioned thermodynamic view [20-22]. This is the birth of the so-called stochastic thermodynamics $[23,24]$. Subsequently, the introduction of the concepts of heat, work and entropy into stochastic dynamics has allowed the principles of thermodynamics to be rediscovered on the basis of the Langevin and Fokker-Planck methodologies [2530]. Furthermore, several fundamental fluctuation theorems have been derived in this context [31-36].

In parallel with the development of stochastic thermodynamics, the remarkable history of non-equilibrium thermodynamics must be outlined [37, 38]. In this case, the macroscopic thermodynamics of systems at equilibrium has been extended to slightly non-equilibrium states. The second principle of thermodynamics is crucial to this development. In fact, to generalize thermodynamics to non-equilibrium processes, the explicit expression for the entropy production is necessary. In the early thirties, Onsager theory predicted that the rate of entropy production is always given by $\frac{d S_{p}}{d t}=\sum_{i=1}^{N} \mathscr{J}_{i} \mathscr{X}_{i}$, where the $\mathscr{X}_{i}$ are the thermodynamic

\footnotetext{
* stefano.giordano@univ-lille.fr
}

forces and the $\mathscr{J}_{i}$ are the corresponding fluxes. The theory is completed by the linearized phenomenological equations $\mathscr{J}_{i}=\sum_{j=1}^{N} \mathscr{L}_{i j} \mathscr{X}_{j}$, where $\mathscr{L}_{i j}$ are the phenomenological coefficients. Reciprocal relations $\mathscr{L}_{i j}=\mathscr{L}_{j i}$ have been introduced by Onsager on the base of the microscopic reversibility $[39,40]$, and have been extensively experimentally verified (see, for instance, Ref.[41]). Moreover, in the presence of physical variables that are odd under time reversal, the Onsager reciprocal relations must be replaced by the OnsagerCasimir generalized relations [42, 43]. The theory has been further extended by de Groot and Mazur for considering vector and tensor quantities with applications to heat conduction, diffusion, viscosity and electromagnetic phenomena [44, 45]. More recent contributions deal with generalized Onsager reciprocal relations for states far from the equilibrium [46], and the demonstration of the Onsager relations with broken timereversal symmetry [47].

Today, thermodynamic theories play a crucial role in the understanding of several nano-systems and physical phenomena, including macromolecular folding and unfolding [4850], molecular motors [51, 52], muscles behavior [53, 54], adhesion processes [55-57], micro- and nano-heat engines [5860], micromagnetism [61-65], and heat transfer in nanostructures [66-68], just to name a few.

For these reasons, in this work we want to further investigate the explicit relation between the stochastic thermodynamics described by Langevin and Fokker-Planck equations and the non-equilibrium thermodynamics defined by the Onsager formalism. In particular, we want to understand if and how the thermodynamic fluxes and the entropy production calculated with stochastic thermodynamics can coincide with those predicted by Onsager's theory. That is, we would like to find the fluxes as a linear function of the thermodynamic forces (imposed by a given protocol), and the entropy production as a quadratic function of the same forces (at least for small forces). We would also like both of these functions to be defined by means of the same phenomenological coefficients, also satisfying the Onsager reciprocal relations.

As a matter of fact, there are many works in the literature dealing with the calculation of the entropic production and/or the Onsager coefficients for stochastic systems of different types and under different thermodynamic conditions, as dis- 


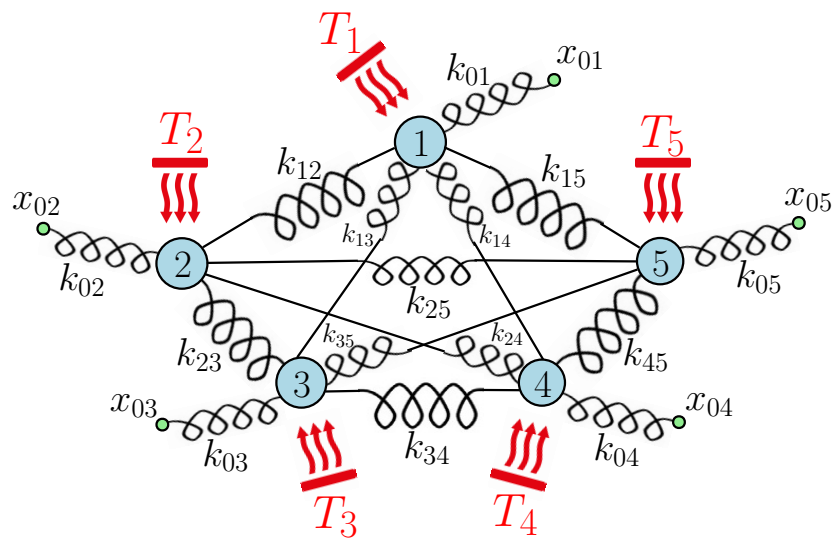

FIG. 1. Example of the harmonic system with $N=5$ particles and with interactions described by a complete graph. The elastic constants $k_{0 i}$ link each point particle $i$ with the fixed point $x_{0 i}$. Moreover, the elastic constants $k_{i j}=k_{j i}$ link the point particles $i$ and $j$. Each particle is in contact with a reservoir at temperature $T_{i}$.

cussed below.

A widely studied situation corresponds to the stationary regime. Concerning linear Langevin systems [69, 70], the possible irreversibility of fluctuations in non-equilibrium steady states was first recognized by Lax [71]. More recently, the reversible and irreversible stationary states of OrnsteinUhlenbeck processes have been further investigated [72], and several expressions for the entropy production have been elaborated [73]. Importantly, in these expressions both even and odd variables under time reversal are considered. Moreover, the meaning of the thermodynamic forces in close-toequilibrium media has been recently clarified in Ref.[74], where the authors considered the structure of the McLennan steady nonequilibrium ensemble [75]. These approaches have been generalized to the study of linear quantum systems as well $[76,77]$. Also the study of the entropy production for non-Markovian dynamical systems has been recently addressed [78, 79]. Finally, expressions for the entropy production in irreversible thermodynamics with friction have been established [80-82].

Another complementary line of research concerns periodically driven stochastic system [83]. Entropy production, Onsager coefficients and efficiency have been determined for a Brownian Carnot cycle and other periodic micro- and nanoheat engines [84-86]. Then, a general theory for the linear stochastic thermodynamics of periodically driven Markovian systems has been developed by considering all Fourier components of the involved quantities $[87,88]$. Also, systems with spatially periodic potentials or sequentially exposed to different reservoirs have been investigated to better understand the implications of the Onsager reciprocity relations [8991]. Fluctuation theorems have been used to bound the thermodynamic currents in periodically driven systems [92, 93]. And finally, entropy production, heat capacity and heat transport properties are studied in harmonic systems under timedependent periodic drivings [94, 95].

In general, the entropic production rate is defined through integrals involving the probability currents [see, e.g., Eqs.(15) and (62), for systems with 1 or $N$ degrees of freedom], which are difficult to calculate for most physical systems and nonequilibrium states. However, their calculation becomes accessible when the system is linear or when we work under stationary or periodic conditions. In the latter two cases, the entropic production is opposite to the entropic flow, and therefore it can be determined from the heat flows in the system. These approaches have been largely developed and generalized in all the above-mentioned works. Here, as a complement to previous efforts, we limit our analysis to a linear system but we work in a non-equilibrium regime, characterized by the relaxation to the thermodynamic equilibrium. The idea is to show that we can retrieve the structure of the Onsager theory during this relaxation. First of all, for pedagogical purpose, we introduce a very simple linear system with one degree of freedom to show the identification of thermodynamic fluxes and forces with the characteristic quantities of stochastic models. In a second step, we consider a linear system composed of $N$ particles described by a harmonic interaction efficiently represented by a complete graph. The coefficients of the graph represent the elastic constants and are completely arbitrary. This means that there is no a priori symmetry in the system. The particles are in contact with $N$ reservoirs with different variable temperatures $T_{i}$ (see Fig.1). In the initial phase, the temperatures are constant in time and different from each other so that the system can reach a non-equilibrium stationary state (NESS), characterized by constant heat flows among particles and a continuous entropy production. At a certain point, the temperatures of the thermal baths are instantaneously set to the same value $T$, thus inducing the relaxation of the system towards equilibrium. It is precisely at this stage that we can identify the thermodynamic forces and fluxes and observe whether the structure of Onsager's theory is respected. For an arbitrary non-equilibrium regime (namely, arbitrary jumps $T-T_{i}$ ), we are able to obtain the linear relation between fluxes and forces, described by symmetric Onsager coefficients. We also provide an explicit expression of these coefficients in terms of the elastic constants characterizing the complete graph of the interactions. Again in the case with arbitrary jumps $T-T_{i}$, we find a rate of entropy production that is nonlinear in thermodynamic forces (similarly to Ref.[77], dealing with open bosonic Gaussian systems), thus representing a nonlinear generalization of the Onsager theory. Only when the jumps $T-T_{i}$ are sufficiently small, the rate of entropy production becomes equal to the classical Onsager quadratic form. This analysis allows a deeper understanding of the mathematical structure of the Onsager theory with arbitrary forces, and is useful to elucidate the relation between stochastic thermodynamics and non-equilibrium thermodynamics.

\section{AN INTRODUCTORY ONE-DIMENSIONAL EXAMPLE}

To begin with, we consider a simple example useful to introduce the idea of relaxation to equilibrium in stochastic thermodynamics. We suppose to observe the one-dimensional motion of a particle (of mass $m$ ) subjected to an elastic force 
(with spring constant $k$ ), to an external force $f$, and embedded in a Langevin heat bath at temperature $T$. The equation of motion can be written in the following form

$$
\begin{aligned}
\dot{x} & =\frac{p}{m}, \\
\dot{p} & =-k x+f-\beta p+\sqrt{k_{B} m \beta T} n(t),
\end{aligned}
$$

where $x$ and $p$ are position and momentum of the particle, respectively. Moreover, $\beta$ is the friction coefficient (or collision frequency), $k_{B}$ is the Boltzmann constant and $n(t)$ is a Gaussian stochastic process with average value $\langle n(t)\rangle=0 \forall t$, and correlation $\left\langle n\left(t_{1}\right) n\left(t_{2}\right)\right\rangle=2 \delta\left(t_{1}-t_{2}\right) \forall t_{1}$ and $t_{2}[16,17]$. Here, the average values are calculated over the noise statistics. In order to consider an arbitrary out-of-equilibrium evolution, we assume arbitrary functions $T=T(t)$ and $f=f(t)$, applied to the system. For the sake of simplicity, and to minimise non-essential difficulties, we consider an overdamped system $(|\dot{p}| \ll \beta|p|)$, where the inertial effects can be neglected as follows

$$
\dot{x}=-\frac{k}{m \beta} x+\frac{f}{m \beta}+\sqrt{\frac{k_{B} T}{m \beta}} n(t) .
$$

The properties of this first order Langevin equation can be studied through the associated Fokker-Planck equation, describing the dynamics of the density probability $W(x, t)$. In the overdamped case it is typically named Smoluchowski equation and takes the form

$$
\frac{\partial W}{\partial t}=\frac{\partial}{\partial x}\left(\frac{k}{m \beta} x W\right)-\frac{\partial}{\partial x}\left(\frac{f}{m \beta} W\right)+\frac{k_{B} T}{m \beta} \frac{\partial^{2} W}{\partial x^{2}},
$$

or equivalently,

$$
\frac{\partial W}{\partial t}=-\frac{\partial J}{\partial x}
$$

where we introduced the quantity

$$
J=-\frac{k}{m \beta} x W+\frac{f}{m \beta} W-\frac{k_{B} T}{m \beta} \frac{\partial W}{\partial x} .
$$

We underline that Eq.(4) represents a one-dimensional continuity equation for the density probability. Despite the simplicity of this system, we show now that it is sufficient to reconstruct the two classical principles of thermodynamics.

We start by describing the first principle, stating the energy balance for the system. If we look at the underdamped version in Eq.(1), we can simply define the total internal energy as $E=\frac{1}{2} k x^{2}+\frac{1}{2} \frac{p^{2}}{m}$. However, if we consider the overdamped version in Eq.(2), we have to redefine the total energy as $E=\frac{1}{2} k x^{2}$, where the kinetic energy is not considered because of the absence of inertia. Consistently, the rate of average internal energy can be elaborated as follows

$$
\begin{aligned}
\frac{d}{d t}\langle E\rangle & =\frac{d}{d t}\left\langle\frac{1}{2} k x^{2}\right\rangle=\frac{d}{d t} \int_{-\infty}^{+\infty} \frac{1}{2} k x^{2} W d x \\
& =\int_{-\infty}^{+\infty} \frac{1}{2} k x^{2} \frac{\partial W}{\partial t} d x=-\int_{-\infty}^{+\infty} \frac{1}{2} k x^{2} \frac{\partial J}{\partial x} d x,
\end{aligned}
$$

where we can now use an integration by parts in the form

$$
\int_{-\infty}^{+\infty} \phi \frac{\partial \psi}{\partial x} d x=-\int_{-\infty}^{+\infty} \psi \frac{\partial \phi}{\partial x} d x .
$$

In Eq.(6), the average value is calculated with respect to the density $W(x, t)$ (we adopted the same symbol but is not the same average as that of noise $n$ above). So doing, we simply obtain

$$
\begin{aligned}
\frac{d}{d t}\langle E\rangle & =\int_{-\infty}^{+\infty} J k x d x \\
& =\int_{-\infty}^{+\infty} J(k x-f) d x+\int_{-\infty}^{+\infty} J f d x,
\end{aligned}
$$

where in the last line we added and subtracted the same quantity representing the integral of $J f$. This quantity corresponds to the rate of work done on the system by the external force $f$. It can be formally proved as follows

$$
\begin{aligned}
\int_{-\infty}^{+\infty} J f d x & =\int_{-\infty}^{+\infty}\left(-\frac{k}{m \beta} x W+\frac{f}{m \beta} W-\frac{k_{B} T}{m \beta} \frac{\partial W}{\partial x}\right) f d x \\
& =\left(-\frac{k}{m \beta}\langle x\rangle+\frac{f}{m \beta}\right) f .
\end{aligned}
$$

Then, by averaging the overdamped Langevin equation stated in Eq.(2), we get $\langle\dot{x}\rangle=\frac{1}{m \beta}(f-k\langle x\rangle)$, and therefore we have that

$$
\int_{-\infty}^{+\infty} J f d x=f\langle\dot{x}\rangle
$$

which exactly corresponds the the rate of work done on the system by the force $f$. Moreover, the first term in the second line of Eq.(8) represents the heat rate entering the system. We have finally obtained the first principle of thermodynamics in the form

$$
\frac{d}{d t}\langle E\rangle=\frac{d}{d t}\langle Q\rangle+\frac{d}{d t}\langle L\rangle,
$$

where the heat rate is $\frac{d}{d t}\langle Q\rangle=\int_{-\infty}^{+\infty} J(k x-f) d x$ and the rate of work is $\frac{d}{d t}\langle L\rangle=\int_{-\infty}^{+\infty} J f d x=f\langle\dot{x}\rangle$.

To further substantiate the expression of the heat rate, we study the time variation of the total entropy of the system, eventually constructing the second principle. Firstly, we introduce the entropy as follows

$$
S=-k_{B} \int_{-\infty}^{+\infty} W \log W d x
$$

and we perform its time derivative

$$
\begin{aligned}
\frac{d S}{d t} & =-k_{B} \int_{-\infty}^{+\infty} \frac{\partial W}{\partial t} \log W d x=k_{B} \int_{-\infty}^{+\infty} \frac{\partial J}{\partial x} \log W d x \\
& =-k_{B} \int_{-\infty}^{+\infty} J \frac{\partial \log W}{\partial x} d x=-k_{B} \int_{-\infty}^{+\infty} J \frac{1}{W} \frac{\partial W}{\partial x} d x
\end{aligned}
$$

Then, we can substitute $\frac{\partial W}{\partial x}$ obtained from Eq.(5), and we get

$$
\begin{aligned}
\frac{d S}{d t} & =-k_{B} \int_{-\infty}^{+\infty} J \frac{1}{W}\left(-\frac{m \beta}{k_{B} T} J-\frac{k}{k_{B} T} x W+\frac{f}{k_{B} T} W\right) d x \\
& =\frac{1}{T} \int_{-\infty}^{+\infty} J(k x-f) d x+\frac{1}{T} \int_{-\infty}^{+\infty} \frac{m \beta}{W} J^{2} d x
\end{aligned}
$$


where the first term represents the entropy flow generated by the heat transfer between thermal bath and system and the second term represents the entropy production generated by the irreversible character of the process. We can indeed write

$$
\frac{d S}{d t}=\frac{1}{T} \frac{d\langle Q\rangle}{d t}+\frac{d S_{p}}{d t}
$$

which represents the second principle of thermodynamics, where we introduced the entropy production rate as

$$
\frac{d S_{p}}{d t}=\frac{1}{T} \int_{-\infty}^{+\infty} \frac{m \beta}{W(x, t)} J(x, t)^{2} d x .
$$

The positive character of this quantity is perfectly consistent with the second principle of thermodynamics. The technique, here presented for obtaining the two thermodynamic principles from the Langevin overdamped equation for a onedimensional system, has been extended to the underdamped case with an arbitrary number of particles [26-30], and to holonomic systems with an arbitrary number of generalized coordinates as well $[96,97]$.

We define now specific conditions on the system to prove that the formalism of the stochastic thermodynamics (based on the Langevin and Fokker-Planck equations and on their further developments) is able to reproduce the structure of the classical non-equilibrium thermodynamics (based on the Onsager theory). We suppose that the system is at thermodynamic equilibrium for $t<0$ with $f=f_{1}$ and $T=T_{1}$. Then, for $t=0$, we suddenly change these values to $f=f_{2}$ and $T=T_{2}$, and we observe the relaxation for $t \geq 0$ of the system to the new thermodynamic equilibrium. The mathematical analysis of this problem is very simple since the Langevin equation stated in Eq.(2) is linear and results in an OrnsteinUhlenbeck stochastic process $[69,70]$. In spite of the simplicity of this problem, we want to stress here that the relaxation to the thermodynamic equilibrium can be represented by the formalism of the classical nonequilibrium thermodynamics where, in general, the entropy production is given by $\frac{d S_{p}}{d t}=\sum_{i=1}^{N} \mathscr{J}_{i} \mathscr{X}_{i}$ where the $\mathscr{X}_{i}$ are the so-called thermodynamic forces and the $\mathscr{J}_{i}$ are the fluxes described by the linearized phenomenological equations $\mathscr{J}_{i}=\sum_{j=1}^{N} \mathscr{L}_{i j} \mathscr{X}_{j}$, with $\mathscr{L}_{i j}$ being the phenomenological coefficients [37]. These coefficients, in absence of an external magnetic field, fulfill the Onsager reciprocal relations $\mathscr{L}_{i j}=\mathscr{L}_{j i}[39,40]$. In our case, we deal with a simple linear one-dimensional system, where forces and fluxes are uncoupled, and therefore we cannot observe this reciprocal property. However, in a following section we will discuss a $N$-dimensional system with coupled forces and fluxes.

At equilibrium, for $t<0$, the average value $\langle x\rangle_{0}$ of the position is simply given

$$
\langle x\rangle_{0}=\frac{f_{1}}{k},
$$

and its variance $\Sigma_{0}=\left\langle\left(x-\langle x\rangle_{0}\right)^{2}\right\rangle$ is given by

$$
\Sigma_{0}=\frac{k_{B} T_{1}}{k}
$$

as one can easily prove, i.e., by means of the OrnsteinUhlenbeck theory $[69,70]$, or by the equilibrium Gibbs distribution. Since the system is linear, the probability density is Gaussian and takes the explicit form

$$
W_{0}(x)=\sqrt{\frac{k}{2 \pi k_{B} T_{1}}} e^{-\frac{1}{2}\left(x-\frac{f_{1}}{k}\right)^{2} \frac{k}{k_{B} T_{1}}},
$$

which is valid for any $t<0$. For $t \geq 0$, the evolution of $\langle x\rangle$ and $\Sigma=\left\langle(x-\langle x\rangle)^{2}\right\rangle$ is governed by the equations $[69,70]$

$$
\begin{aligned}
\frac{d\langle x\rangle}{d t} & =-\frac{k}{m \beta}\langle x\rangle+\frac{f_{2}}{m \beta}, \\
\frac{d \Sigma}{d t} & =-\frac{2 k}{m \beta} \Sigma+2 \frac{k_{B} T_{2}}{m \beta},
\end{aligned}
$$

with solutions

$$
\begin{aligned}
\langle x\rangle & =\frac{f_{1}-f_{2}}{k} e^{-\frac{k}{m \beta} t}+\frac{f_{2}}{k}, \\
\Sigma & =k_{B} \frac{T_{1}-T_{2}}{k} e^{-\frac{2 k}{m \beta} t}+\frac{k_{B} T_{2}}{k} .
\end{aligned}
$$

Of course, also for $t \geq 0$, the probability density $W(x, t)$ is Gaussian,

$$
W(x, t)=\sqrt{\frac{1}{2 \pi \Sigma}} e^{-\frac{1}{2 \Sigma}(x-\langle x\rangle)^{2}},
$$

with average value and variance given in Eqs.(21) and (22). Of course, we have $W(x, 0)=W_{0}(x)$. The relaxation to the new thermodynamic equilibrium generated by the values $f_{2}$ and $T_{2}$ can be described by the fluxes $\mathscr{J}_{1}=\frac{d\langle x\rangle}{d t}$ and $\mathscr{J}_{2}=\frac{d\langle Q\rangle}{d t}$, representing the average velocity of the particle and the heat rate exchanged with the thermal bath, respectively. Indeed, the particle must change its position and its average kinetic energy to attain the new thermodynamic equilibrium. For $t \geq$ 0 , the first flux is directly obtained from Eq.(21), as follows

$$
\mathscr{J}_{1}=\frac{d\langle x\rangle}{d t}=\frac{f_{2}-f_{1}}{m \beta} e^{-\frac{k}{m \beta} t}
$$

The second flux can be calculated through the expression

$$
\mathscr{J}_{2}=\frac{d\langle Q\rangle}{d t}=\int_{-\infty}^{+\infty} J\left(k x-f_{2}\right) d x
$$

where $J$ is the flux of the Smoluchowski equation

$$
J=-\frac{k}{m \beta} x W+\frac{f_{2}}{m \beta} W-\frac{k_{B} T_{2}}{m \beta} \frac{\partial W}{\partial x} .
$$

Here, we considered $f_{2}$ and $T_{2}$ in Eq.(5) since we are working for time $t>0$. The substitution of Eq.(26) in Eq.(25) leads to

$$
\mathscr{J}_{2}=2 \frac{f_{2} k}{m \beta}\langle x\rangle-\frac{k^{2}}{m \beta}\left(\Sigma+\langle x\rangle^{2}\right)+k \frac{k_{B} T_{2}}{m \beta}-\frac{f_{2}^{2}}{m \beta},
$$

where we can use Eqs.(21) and (22) to get the final result for $\mathscr{J}_{2}$ in the form

$$
\mathscr{J}_{2}=\frac{d\langle Q\rangle}{d t}=k \frac{k_{B}\left(T_{2}-T_{1}\right)}{m \beta} e^{-\frac{2 k}{m \beta} t}-\frac{\left(f_{2}-f_{1}\right)^{2}}{m \beta} e^{-\frac{2 k}{m \beta} t} .
$$


In order to have a complete picture of the system evolution for $t \geq 0$, it is important to obtain an explicit expression for the quantities entering the second principle of thermodynamics, as stated in Eq.(14). First of all, we consider the total entropy, and for the Gaussian distribution defined in Eq.(23), we have

$$
S=-k_{B} \int_{-\infty}^{+\infty} W \log W d x=\frac{k_{B}}{2} \log (2 \pi e \Sigma) .
$$

Hence, we can calculate the total entropy rate during the relaxation to the equilibrium through the following derivative

$$
\frac{d S}{d t}=\frac{k_{B}}{2} \frac{1}{\Sigma} \frac{d \Sigma}{d t}=-\frac{k}{m \beta} \frac{k_{B}\left(T_{1}-T_{2}\right) e^{-\frac{2 k}{m \beta} t}}{\left(T_{1}-T_{2}\right) e^{-\frac{2 k}{m \beta} t}+T_{2}},
$$

where we introduced the expression of the variance given in Eq.(22). The entropy flow is simply given by $\frac{1}{T} \frac{d\langle Q\rangle}{d t}$ and therefore it is sufficient to divide by $T$ the heat flow given in Eq.(28). To conclude, we consider the entropy production rate. It can be calculated either by its definition in Eq.(15), or by using the second principle stated in Eq.(14). Both procedures lead to the following result

$$
\begin{aligned}
\frac{d S_{p}}{d t}= & \frac{1}{T_{2}} \int_{-\infty}^{+\infty} \frac{m \beta}{W} J^{2} d x=\frac{d S}{d t}-\frac{1}{T_{2}} \frac{d\langle Q\rangle}{d t}= \\
= & \frac{k}{m \beta} k_{B}\left(\frac{T_{2}-T_{1}}{T_{2}}\right)^{2} \frac{e^{-\frac{4 k}{m \beta} t}}{1-\frac{T_{2}-T_{1}}{T_{2}} e^{-\frac{2 k}{m \beta} t}} \\
& +\frac{1}{m \beta}\left(f_{2}-f_{1}\right)^{2} \frac{1}{T_{2}} e^{-\frac{2 k}{m \beta} t} .
\end{aligned}
$$

With the aim of reconciling these results of the stochastic thermodynamics with the known structure of out-ofequilibrium thermodynamics, we consider a small deviation between the initial state and the final state. It means that we assume $\delta T=T_{2}-T_{1}$ and $\delta f=f_{2}-f_{1}$ as arbitrarily small quantities, and we rewrite the fluxes $\mathscr{J}_{1}$ and $\mathscr{J}_{2}$ to the first order in $\delta T$ and $\delta f$. We simply get

$$
\begin{aligned}
& \mathscr{J}_{1}=\frac{1}{m \beta} \delta f e^{-\frac{k}{m \beta} t} \\
& \mathscr{J}_{2}=k \frac{k_{B}}{m \beta} \delta T e^{-\frac{2 k}{m \beta} t} .
\end{aligned}
$$

We observe that the second term in Eq.(28) is not considered in Eq.(33) since is of the second order in $\delta f$. This shows that stochastic thermodynamics can provide higher order information than non-equilibrium thermodynamics. We also underline that Eqs.(32) and (33) represent a first form of the phenomenological equation mapping thermodynamic fluxes and forces. To better understand the structure of the nonequilibrium thermodynamics, we rewrite the entropy production rate under the same assumptions, as follows

$$
\begin{aligned}
\frac{d S_{p}}{d t} & =\frac{k}{m \beta} k_{B}\left(\frac{\delta T}{T_{2}}\right)^{2} e^{-\frac{4 k}{m \beta} t}+\frac{1}{m \beta}(\delta f)^{2} \frac{1}{T_{2}} e^{-\frac{2 k}{m \beta} t} \\
& =\mathscr{J}_{1} \mathscr{X}_{1}+\mathscr{J}_{2} \mathscr{X}_{2},
\end{aligned}
$$

where we introduced the thermodynamic forces

$$
\begin{aligned}
& \mathscr{X}_{1}=\frac{\delta f}{T_{2}} e^{-\frac{k}{m \beta} t}, \\
& \mathscr{X}_{2}=\frac{\delta T}{T_{2}^{2}} e^{-\frac{2 k}{m \beta} t} .
\end{aligned}
$$

So doing, the linear phenomenological equations for the system are in the form $\mathscr{J}_{1}=\frac{T_{2}}{m \beta} \mathscr{X}_{1}$ and $\mathscr{J}_{2}=k \frac{k_{B} T_{2}^{2}}{m \beta} \mathscr{X}_{2}$. It means that $\mathscr{L}_{11}=\frac{T_{2}}{m \beta}, \mathscr{L}_{22}=k \frac{k_{B} T_{2}^{2}}{m \beta}$, and $\mathscr{L}_{12}=\mathscr{L}_{21}=0$. Moreover, the rate of entropy production assumes the classical quadratic form $\frac{d S_{p}}{d t}=\mathscr{L}_{11} \mathscr{X}_{1}^{2}+\mathscr{L}_{22} \mathscr{X}_{2}^{2}$. We have that $\mathscr{L}_{12}=\mathscr{L}_{21}=0$ since the system is linear. This is a very simple example but is useful to understand that the expression of the entropy production rate given in Eq.(15) is perfectly compatible with the structure of the out-of-equilibrium thermodynamics. It is worth noticing that this structure can be also recognized by simply observing the system for $t=0$. Indeed, the deviations $\delta f$ and $\delta T$ are able to instantaneously generate thermodynamic forces and the corresponding fluxes, when applied to the system. It means that it is not necessary to solve the equations describing average values and covariances during the relaxation to the equilibrium to identify the phenomenological coefficients. This point is useful to study more complex systems, as discussed in the next Section.

\section{OVERDAMPED N-DIMENSIONAL SYSTEM}

In this Section, we consider a system described by $N$ degrees of freedom, which is at NESS for $t<0$, and that shows a relaxation to equilibrium for $t \geq 0$. The system is harmonic and governed by overdamped Langevin equations with $N$ different thermal baths. This assumption allows us to use the results of the Ornstein-Uhlenbeck theory. We prove that the relaxation to the equilibrium can be described by a linear map between thermodynamic fluxes and forces, with phenomenological coefficients satisfying the Onsager reciprocal relations. Moreover, for small forces, the rate of entropy production can be written as a quadratic form in the thermodynamic forces, defined through the same set of phenomenological coefficients.

\section{A. System definition}

We consider an elastic network composed of $N$ point particles with positions $\vec{x}=\left(x_{1}, \ldots, x_{N}\right) \in \mathbb{R}^{N}$ on the $x$-axis and interacting through the potential energy

$$
V(\vec{x})=\frac{1}{2} \sum_{i=1}^{N} k_{0 i}\left(x_{i}-x_{0 i}\right)^{2}+\frac{1}{4} \sum_{i=1}^{N} \sum_{j=1}^{N} k_{i j}\left(x_{i}-x_{j}\right)^{2},
$$

stating that each point particle $i$ is connected with all the others $j \neq i$ and with a fixed point $x_{0 i}$. The elastic constants $k_{0 i}>0$ link each point particle $i$ with the fixed point $x_{0 i}$. Moreover, the elastic constants $k_{i j}=k_{j i}>0$ (with $k_{n n}=0 \forall n=1, \ldots, N$ ) 
link the point particles $i$ and $j$ over the complete graph describing the elastic network (see Fig.1). We underline that this is the most complex case of elastic interaction among the $N$ particles. The network, or equivalently the complete graph, is arbitrarily heterogeneous. In addition, we suppose that all the point particles have a mass $m$ and that they are embedded in thermal baths with the same collision frequency $\beta$ but with different temperatures $T_{i}$. We adopted the same values $m$ and $\beta$ for all point particles for the sake of simplicity but all the following results could be also obtained with heterogeneous masses and collisions frequencies. We underline that a nonequilibrium thermodynamic approach for complex networks or graphs is developed in Ref.[98] from a different point of view. The overdamped dynamics of our system is described by the following system of stochastic differential equations

$$
\dot{x}_{i}=-\frac{1}{m \beta} \frac{\partial V(\vec{x})}{\partial x_{i}}+\sqrt{\frac{k_{B} T_{i}}{m \beta}} n_{i}(t),
$$

where the elastic forces can be determined by deriving the potential energy

$$
-\frac{\partial V(\vec{x})}{\partial x_{i}}=-k_{0 i}\left(x_{i}-x_{0 i}\right)-\sum_{j=1}^{N} k_{i j}\left(x_{i}-x_{j}\right),
$$

and where the noises satisfy the relations $\left\langle n_{i}(t)\right\rangle=0$ and $\left\langle n_{i}\left(t_{1}\right) n_{j}\left(t_{2}\right)\right\rangle=2 \delta_{i j} \delta\left(t_{1}-t_{2}\right)[16,17]$. In this Section we study the temperature-related thermodynamic forces but to simplify the analysis we ignore the displacement-related thermodynamic forces. It means that, unlike the previous Section, we do not take into consideration mechanical forces applied to the particles. This assumption also stems from the fact that in linear systems the thermal and mechanical effects are uncoupled and thus the corresponding Onsager cross-coefficients are zero. Since we are interested in studying Onsager relations in stochastic thermodynamics, we restrict ourselves to the purely thermal case.

We first analyze the general properties of the out-ofequilibrium evolution of the system in the most general case with arbitrarily time-varying thermostats $T_{i}=T_{i}(t)$. To carry out this analysis, the main system of equations can be rewritten in matrix form as follows

$$
\frac{d \vec{x}}{d t}=-\frac{1}{m \beta}\left(\mathbb{K} \vec{x}-\vec{f}_{0}\right)+\mathbb{G} \vec{n}(t),
$$

where we introduced the vectors $\left(\vec{f}_{0}\right)_{i}=k_{0 i} x_{0 i}$ and $(\vec{n})_{i}=n_{i}$ and the matrices $\mathbb{G}=\operatorname{diag}\left(\sqrt{\frac{k_{B} T_{i}}{m \beta}}\right)$ and

$$
\mathbb{K}=\left[\begin{array}{cccc}
k_{01}+\sum_{j \neq 1} k_{1 j} & -k_{12} & -k_{13} & \ldots \\
-k_{12} & k_{02}+\sum_{j \neq 2} k_{2 j} & -k_{23} & \ldots \\
-k_{13} & -k_{23} & k_{03}+\sum_{j \neq 2} k_{3 j} & \ldots \\
\vdots & \vdots & \vdots & \ddots
\end{array}\right] .
$$

Here, $\operatorname{diag}\left(a_{i}\right)$ represents a diagonal matrix with the elements $a_{i}$ on the main diagonal $(i=1, \ldots, N)$. This is a standard
Ornstein-Uhlenbeck process $[69,70]$, and therefore the average value of positions $\langle\vec{x}\rangle$ and the covariance matrix $\mathbb{\Sigma}=$ $\left\langle(\vec{x}-\langle\vec{x}\rangle)(\vec{x}-\langle\vec{x}\rangle)^{T}\right\rangle$ are described by the following relations

$$
\begin{aligned}
\frac{d\langle\vec{x}\rangle}{d t} & =-\frac{1}{m \beta}\left(\mathbb{K}\langle\vec{x}\rangle-\vec{f}_{0}\right), \\
\frac{d \mathbb{Z}}{d t} & =-\frac{1}{m \beta}(\mathbb{K} \mathbb{Z}+\mathbb{Z} \mathbb{K})+2 \mathbb{G} \mathbb{G}^{T} .
\end{aligned}
$$

We observe that in a possible NESS regime we have $\langle\vec{x}\rangle=$ $\mathbb{K}^{-1} \vec{f}_{0}$ and then we define $\vec{x}^{s s}=\mathbb{K}^{-1} \vec{f}_{0}$. We remark that the NESS can be attained only if all the thermal bath temperatures are constant over time. Nevertheless, we can always define $\vec{x}^{s s}=\mathbb{K}^{-1} \vec{f}_{0}$ or $\vec{f}_{0}=\mathbb{K} \vec{x}^{s s}$. Anyway, the Fokker-Planck methodology can be applied to Eq.(40) and yields the following Smoluchovski equation for the overdamped problem under investigation

$$
\frac{\partial W}{\partial t}=\frac{1}{m \beta} \frac{\partial}{\partial \vec{x}}\left[\left(\mathbb{K} \vec{x}-\vec{f}_{0}\right) W\right]+\frac{\partial}{\partial \vec{x}}\left(\mathbb{G}^{T} \frac{\partial W}{\partial \vec{x}}\right) .
$$

By the introduction of the flux

$$
\vec{J}=-\frac{1}{m \beta}\left(\mathbb{K} \vec{x}-\vec{f}_{0}\right) W-\mathbb{G G}^{T} \frac{\partial W}{\partial \vec{x}},
$$

the Smoluchovski equation can be rewritten as

$$
\frac{\partial W}{\partial t}=-\frac{\partial \vec{J}}{\partial \vec{x}}
$$

which represents a standard continuity equation. As it is well known in the theory of the Ornstein-Ulhenbeck theory [69, 70], the general solution of Eq.(44) can be obtained in the following Gaussian form

$$
W(\vec{x}, t)=\frac{1}{\sqrt{(2 \pi)^{N} \operatorname{det} \mathbb{\Sigma}}} \exp \left[-\frac{1}{2}(\vec{x}-\langle\vec{x}\rangle)^{T} \mathbb{Z}^{-1}(\vec{x}-\langle\vec{x}\rangle)\right],
$$

completely defined by the average value $\langle\vec{x}\rangle$ and by the covariance matrix $\mathbb{Z}$, described by Eqs.(42) and (43).

\section{B. Thermodynamic principles}

We determine now the mathematical form of the two thermodynamic principles for the system under investigation.

Concerning the first principle we observe that the total energy of the system is simply constituted by the potential energy because of the overdamped motion assumption, as discussed in Section II. Therefore, we can directly obtain the expression

$$
\begin{aligned}
\frac{d}{d t}\langle E\rangle & =\frac{d}{d t}\langle V\rangle=\int_{\mathbb{R}^{N}} V \frac{\partial W}{\partial t} d \vec{x} \\
& =-\int_{\mathbb{R}^{N}} V \frac{\partial \vec{J}}{\partial \vec{x}} d \vec{x}=\int_{\mathbb{R}^{N}} \vec{J} \cdot \frac{\partial V}{\partial \vec{x}} d \vec{x} .
\end{aligned}
$$


Since in this case we have no external forces applied to the system, the right hand side of Eq.(48) must corresponds to the total heat rate entering the system. Hence we can write

$$
\frac{d}{d t}\langle E\rangle=\frac{d}{d t}\langle Q\rangle=\sum_{i=1}^{N} \frac{d}{d t}\left\langle Q_{i}\right\rangle,
$$

where we can calculate the heat rate pertaining to the point particle $i$ through the expression

$$
\frac{d\left\langle Q_{i}\right\rangle}{d t}=\int_{\mathbb{R}^{N}} J_{i} \frac{\partial V}{\partial x_{i}} d \vec{x},
$$

without implicit sum over the index $i$.

This result can be further confirmed by developing the second principle of thermodynamics, as follows. We define the entropy through the natural generalization of Eq.(11), i.e. by the expression $S=-k_{B} \int_{\mathbb{R}^{N}} W \log W d \vec{x}$, and then, by performing straightforward calculations as in Eq.(12), we find

$$
\frac{d S}{d t}=-k_{B} \int_{\mathbb{R}^{N}} \frac{1}{W} \vec{J} \cdot \frac{\partial W}{\partial \vec{x}} d \vec{x} .
$$

Here, we can substitute $\frac{\partial W}{\partial x_{i}}$ obtained from Eq.(45), i.e.

$$
\frac{\partial W}{\partial x_{i}}=-\frac{m \beta}{k_{B} T_{i}}\left(J_{i}+\frac{1}{m \beta} W \frac{\partial V}{\partial x_{i}}\right),
$$

finally obtaining the second principle in the form

$$
\frac{d S}{d t}=\sum_{i=1}^{N} \frac{1}{T_{i}} \int_{\mathbb{R}^{N}} J_{i} \frac{\partial V}{\partial x_{i}} d \vec{x}+\sum_{i=1}^{N} \frac{m \beta}{T_{i}} \int_{\mathbb{R}^{N}} \frac{J_{i}^{2}}{W} d \vec{x},
$$

or, in the more classical form

$$
\frac{d S}{d t}=\sum_{i=1}^{N} \frac{1}{T_{i}} \frac{d\left\langle Q_{i}\right\rangle}{d t}+\frac{d S_{p}}{d t}
$$

where the first sum represents the entropy flow associated to the heat fluxes [see also Eq.(50)], and the second term the entropy production related to the irreversible character of the process.

For later use, we determine a specific expression for the three terms entering the second principle for the system under investigation. Let's start with the first one, $\frac{d S}{d t}$, representing the total entropy rate during the system evolution. By considering again the definition $S=-k_{B} \int_{\mathbb{R}^{N}} W \log W d \vec{x}$, we can take into account the Gaussian distribution stated in Eq.(47) and we get

$$
S=\frac{k_{B}}{2} \log [\operatorname{det}(2 \pi e \mathbb{Z})] .
$$

Then, we can perform the time derivative and we obtain

$$
\frac{d S}{d t}=\frac{k_{B}}{2} \frac{1}{\operatorname{det} \mathbb{\Sigma}} \frac{d \operatorname{det} \mathbb{\Sigma}}{d t}=\frac{k_{B}}{2} \operatorname{tr}\left(\mathbb{\Sigma}^{-1} \frac{d \mathbb{\Sigma}}{d t}\right),
$$

where we have used the standard matrix property $\frac{d \operatorname{det} \Sigma}{d t}=$ $\operatorname{det} \mathbb{Z} \operatorname{tr}\left(\mathbb{Z}^{-1} \frac{d \mathbb{\Sigma}}{d t}\right)$, holding for any non-singular matrix $\mathbb{Z}$.
Since the covariance matrix is governed by Eq.(43) (OrnsteinUhlenbeck theory), we find the total entropy rate as

$$
\begin{aligned}
\frac{d S}{d t} & =k_{B} \operatorname{tr}\left(\mathbb{Z}^{-1} \mathbb{G} \mathbb{G}^{T}-\frac{1}{m \beta} \mathbb{K}\right) \\
& =-\frac{k_{B}}{m \beta} \sum_{i=1}^{N} \mathbb{K}_{i i}+\frac{k_{B}}{m \beta} \sum_{i=1}^{N} k_{B} T_{i}\left(\mathbb{Z}^{-1}\right)_{i i} .
\end{aligned}
$$

The second term entering the second principle represents the entropy flow $\sum_{i=1}^{N} \frac{1}{T_{i}} \frac{d\left\langle Q_{i}\right\rangle}{d t}$, which depends directly on the heat rates $\frac{d\left\langle Q_{i}\right\rangle}{d t}$. These quantities can be naturally calculated by means of Eq.(50). With the help of the Smoluchosky fluxes, written in the form

$$
J_{i}=-\frac{1}{m \beta} \frac{\partial V}{\partial x_{i}} W-\frac{k_{B} T_{i}}{m \beta} \frac{\partial W}{\partial x_{i}},
$$

we easily derive this practical expression for the heat rate associated to each particle

$$
\frac{d\left\langle Q_{i}\right\rangle}{d t}=-\frac{1}{m \beta}\left\langle\left(\frac{\partial V}{\partial x_{i}}\right)^{2}\right\rangle+\frac{k_{B} T_{i}}{m \beta}\left\langle\frac{\partial^{2} V}{\partial x_{i}^{2}}\right\rangle .
$$

Here, the quadratic form of the potential energy $V(\vec{x})$ allows a direct calculation yielding the result

$$
\begin{aligned}
\frac{d\left\langle Q_{i}\right\rangle}{d t}= & -\frac{1}{m \beta}\left\{\mathbb{K}\left[\mathbb{Z}+\left(\langle\vec{x}\rangle-\vec{x}^{s s}\right)\left(\langle\vec{x}\rangle-\vec{x}^{s s}\right)^{T}\right] \mathbb{K}\right\}_{i i} \\
& +\frac{k_{B} T_{i}}{m \beta} \mathbb{K}_{i i},
\end{aligned}
$$

representing the heat rate for any point particle. These expressions can be combined to give the entropy flow for the whole system

$$
\begin{aligned}
& \sum_{i=1}^{N} \frac{1}{T_{i}} \frac{d\left\langle Q_{i}\right\rangle}{d t}=\frac{k_{B}}{m \beta} \sum_{i=1}^{N} \mathbb{K}_{i i} \\
& -\frac{1}{m \beta} \sum_{i=1}^{N} \frac{1}{T_{i}}\left\{\mathbb{K}\left[\mathbb{Z}+\left(\langle\vec{x}\rangle-\vec{x}^{s s}\right)\left(\langle\vec{x}\rangle-\vec{x}^{s s}\right)^{T}\right] \mathbb{K}\right\}_{i i} .
\end{aligned}
$$

Please note that Eqs.(60) and (61) are valid for an arbitrary out-of-equilibrium evolution of the system and the presence of quantity $\vec{x}^{s s}$ does not mean that we are in a steady state. We have simply adopted the definition $\vec{x}^{s s}=\mathbb{K}^{-1} \vec{f}_{0}$, as previously discussed.

The third and final term entering the second principle is represented by the entropy production $\frac{d S_{p}}{d t}$, defined as

$$
\frac{d S_{p}}{d t}=\sum_{i=1}^{N} \frac{m \beta}{T_{i}} \int_{\mathbb{R}^{N}} \frac{J_{i}(\vec{x}, t)^{2}}{W(\vec{x}, t)} d \vec{x} .
$$

In order to develop an explicit expression for this contribution, we use Eq.(58) combined with the formula $\frac{\partial V}{\partial x_{i}}=\mathbb{K}_{i s} x_{s}-\left(\vec{f}_{0}\right)_{i}$ and with the property $\frac{\partial W}{\partial x_{i}}=W\left[\mathbb{Z}^{-1}(\vec{x}-\langle\vec{x}\rangle)\right]_{i}$ [directly coming from Eq.(47)]. Straightforward calculations lead to the result

$$
\begin{aligned}
& \frac{d S_{p}}{d t}=-2 \frac{k_{B}}{m \beta} \sum_{i=1}^{N} \mathbb{K}_{i i}+\frac{k_{B}}{m \beta} \sum_{i=1}^{N} k_{B} T_{i}\left(\mathbb{Z}^{-1}\right)_{i i} \\
& +\frac{1}{m \beta} \sum_{i=1}^{N} \frac{1}{T_{i}}\left\{\mathbb{K}\left[\mathbb{Z}+\left(\langle\vec{x}\rangle-\vec{x}^{s s}\right)\left(\langle\vec{x}\rangle-\vec{x}^{s s}\right)^{T}\right] \mathbb{K}\right\}_{i i} .
\end{aligned}
$$


As a check on the developed procedure, we can immediately observe that the three contributions obtained in Eqs.(57), (61) and (63) identically satisfy the second principle stated in Eq.(54). It is important to observe that the time evolution of the three terms directly depends on the dynamics of the average value $\langle\vec{x}\rangle$ and of the covariance $\mathbb{Z}$.

\section{Relaxation to equilibrium}

We introduce now a particular evolution of the temperatures $T_{i}(t)$, useful to investigate the relation between the stochastic thermodynamics and the non-equilibrium thermodynamics. This is the most important conceptual point of this work. We suppose that the system is at NESS for $t<0$ with constant temperatures $T_{i}(t)=T_{i}$ over time $t<0\left(T_{i} \neq T_{j}\right.$ for $\left.i \neq j\right)$. Then, we assume that all the temperatures instantaneously change to the value $T$ for $t \geq 0$. It means that $T_{i}(t)=T \forall i$ for $t \geq 0$. So doing, we can observe a relaxation to the thermodynamic equilibrium for $t \geq 0$ and it is important to verify that the stochastic thermodynamic is able to give the classical results based on thermodynamic forces and fluxes.

From the one hand, for $t<0$, the NESS regime is attained and therefore $\frac{d\langle\vec{x}\rangle}{d t}=0$ and $\frac{d \mathbb{\Sigma}}{d t}=0$. Hence, by using Eqs.(42) and (43) for $t \leq 0$, we have

$$
\begin{aligned}
& \langle\vec{x}\rangle=\vec{x}^{s s} \text { with } \vec{x}^{s s}=\mathbb{K}^{-1} \vec{f}_{0}, \\
& \mathbb{Z}=\mathbb{\mathbb { Z }}^{s s} \text { with } \mathbb{K} \mathbb{Z}^{s s}+\mathbb{Z}^{s s} \mathbb{K}=2 m \beta \mathbb{G G}^{T},
\end{aligned}
$$

where $\mathbb{G}=\operatorname{diag}\left(\sqrt{\frac{k_{B} T_{i}}{m \beta}}\right)$ and $\mathbb{K}$ is given in Eq.(41). Of course, in this stationary regime, for $t<0$, we have $\frac{d}{d t}\langle E\rangle=0, \frac{d S}{d t}=0$ and $\sum_{i=1}^{N} \frac{d\left\langle Q_{i}\right\rangle}{d t}=0$, as can be easily verified. Moreover the rate of entropy flow is opposed to the rate of entropy production to satisfy the second principle of thermodynamics.

On the other hand, for $t \geq 0$, the system evolves to perform the relaxation to the equilibrium thermodynamics at temperature $T$ (indeed, $T_{i}(t)=T \forall i, \forall t \geq 0$ ). In this situation, for $t \geq 0$, the average value $\langle\vec{x}\rangle$ remains constantly equal to $\vec{x}^{s s}$, as can be easily deduced from Eq.(42) with the initial condition $\langle\vec{x}\rangle(0)=\vec{x}^{s s}$. Moreover, the covariance matrix evolves from the initial value $\mathbb{Z}=\mathbb{Z}^{s s}$ for $t=0$ [defined by Eq.(65)] to the equilibrium value $\mathbb{Z}=k_{B} T \mathbb{K}^{-1}$ for $t \rightarrow \infty$. Indeed, the asymptotic solution of Eq.(43) for $t \rightarrow \infty$ with $T_{i}(t)=T \forall i, \forall t \geq 0$ is exactly given by $\mathbb{Z}=k_{B} T \mathbb{K}^{-1}$ for $t \rightarrow \infty$. Consistently, we remark that the covariance $\mathbb{Z}=k_{B} T \mathbb{K}^{-1}$ corresponds to the Gibbs distribution of the equilibrium statistical mechanics.

We are interested in studying the mathematical form of the heat fluxes and the rate of entropy production during the relaxation to equilibrium. It is worth understanding whether stochastic thermodynamics is consistent with Onsager's nonequilibrium theory. For $t \geq 0$, the heat fluxes are given by Eq.(60), where we substitute $\langle\vec{x}\rangle=\vec{x}^{s s}$ and $T_{i}(t)=T \forall i, \forall t \geq$ 0 . We get

$$
\begin{aligned}
\frac{d\left\langle Q_{i}\right\rangle}{d t} & =-\frac{1}{m \beta}\{\mathbb{K} \mathbb{Z} \mathbb{K}\}_{i i}+\frac{k_{B} T}{m \beta} \mathbb{K}_{i i} \\
& =-\frac{1}{m \beta}\left\{\mathbb{K} \mathbb{Z} \mathbb{K}-k_{B} T \mathbb{K}\right\}_{i i} .
\end{aligned}
$$

Similarly, again for $t \geq 0$, the rate of entropy production is given by Eq.(63) with $\langle\vec{x}\rangle=\vec{x}^{s s}$ and $T_{i}(t)=T \forall i, \forall t \geq 0$. We have

$$
\begin{aligned}
\frac{d S_{p}}{d t} & =-2 \frac{k_{B}}{m \beta} \sum_{i=1}^{N} \mathbb{K}_{i i}+\frac{k_{B}^{2} T}{m \beta} \sum_{i=1}^{N}\left(\mathbb{Z}^{-1}\right)_{i i}+\frac{1}{m \beta T} \sum_{i=1}^{N}\{\mathbb{K} \mathbb{Z} \mathbb{K}\}_{i i} \\
& =\frac{1}{m \beta T} \operatorname{tr}\left\{\mathbb{K} \mathbb{Z} \mathbb{K}-2 k_{B} T \mathbb{K}+k_{B}^{2} T^{2} \mathbb{Z}^{-1}\right\} .
\end{aligned}
$$

We wish to prove that Eqs.(66) and (67) are consistent with the formalism of the non-equilibrium thermodynamics. As it is well known, the formalism of non-equilibrium thermodynamics is valid for small deviations from equilibrium. This suggests to represent the covariance matrix $\mathbb{Z}$ as a perturbation of its asymptotic equilibrium value $k_{B} T K^{-1}$. Hence we define

$$
\mathbb{Z}=k_{B} T \mathbb{K}^{-1}+k_{B} \mathbb{\Delta},
$$

where $\Delta$ represents the perturbation (i.e. $\Delta=0$ at equilibrium, for $t \rightarrow \infty)$. With this representation, Eq.(66) immediately delivers

$$
\frac{d\left\langle Q_{i}\right\rangle}{d t}=-\frac{k_{B}}{m \beta}\{\mathbb{K} \Delta \mathbb{K}\}_{i i} .
$$

As discussed in Section II, in order to investigate the structure of the non-equilibrium thermodynamics, it is sufficient to observe the beginning of the relaxation for $t=0$. As a matter of fact, the temperature jumps $T-T_{i}$ are able to instantaneously generate the thermodynamic forces and the corresponding fluxes, which drive the system to the equilibrium. It means that we can analyze the quantities

$$
\mathscr{J}_{i}=\left.\frac{d\left\langle Q_{i}\right\rangle}{d t}\right|_{t=0}=-\frac{k_{B}}{m \beta}\left\{\mathbb{K} \Delta^{s s} \mathbb{K}\right\}_{i i},
$$

where $\Delta^{s s}=\left.\Delta\right|_{t=0}$ is defined by $\mathbb{Z}^{s s}=k_{B} T \mathbb{K}^{-1}+k_{B} \Delta^{s s}$ and $\mathbb{Z}^{s s}$ by Eq.(65), which characterize the NESS regime. These quantities represent the heat flux at the initial time of the relaxation towards equilibrium. Therefore, they should be proportional to the temperature jumps $\delta T_{i}=T-T_{i}$. Indeed, by substituting $\mathbb{Z}^{s s}=k_{B} T \mathbb{K}^{-1}+k_{B} \Delta^{s s}$ in Eq.(65), we obtain

$$
\mathbb{K} \Delta^{s s}+\Delta^{s s} \mathbb{K}=-2 \operatorname{diag}\left(\delta T_{i}\right)
$$

showing that the perturbation $\Delta^{s s}$ effectively depends on the temperature jumps $\delta T_{i}=T_{i}-T$. We can usefully decompose $\triangle^{s s}$ as

$$
\triangle^{s s}=\sum_{j=1}^{N} \triangle_{j}^{s s} \delta T_{j},
$$

so that any matrix $\Delta_{j}^{s s}$ is solution of the simpler equation

$$
\mathbb{K} \Delta_{j}^{s s}+\triangle_{j}^{s s} \mathbb{K}=-2 \mathbb{E}_{j},
$$

where $\mathbb{E}_{j}$ is the elementary matrix with only one element equal to one in the diagonal position $(j, j)$, and all other elements being zero. In other words, we have that $\left\{\mathbb{E}_{j}\right\}_{n m}=$ $\delta_{j n} \delta_{j m}$. The validity of Eq.(73) can be easily proved by observing that $\operatorname{diag}\left(\delta T_{i}\right)=\sum_{j=1}^{N} \delta T_{j} \mathbb{E}_{j}$. The importance of 
Eq.(73) lies in the fact that it can be solved explicitly in the following way. Since the matrix equation $\mathbb{A} \mathcal{X}+\mathcal{X} \mathbb{A}=\mathbb{C}$ (sometimes called Sylvester or Lyapunov equation, see e.g. Refs.[99-101]) has the unique solution

$$
\mathbb{X}=-\int_{0}^{+\infty} e^{\mathbb{A} \xi} \mathbb{C} e^{\mathbb{A} \xi} d \xi
$$

if $\mathbb{A}$ has all eigenvalues with negative real part, we can write the explicit solution of Eq.(73) as

$$
\mathbb{\Delta}_{j}^{s s}=-2 \int_{0}^{+\infty} e^{-\mathbb{k} \xi} \mathbb{E}_{j} e^{-\mathbb{K} \xi} d \xi .
$$

The matrix $\mathbb{K}$ is in fact positive-definite, being at the origin of the quadratic form that defines the potential energy of an asymptotically stable mechanical system. Now, Eqs.(72) and (75) can be replaced into Eq.(70) to determine the thermodynamic fluxes in terms of the temperature jumps

$$
\begin{aligned}
\mathscr{J}_{i} & =-\frac{k_{B}}{m \beta} \sum_{j=1}^{N}\left\{\mathbb{K} \Delta_{j}^{s s} \mathbb{K}\right\}_{i i} \delta T_{j} \\
& =\frac{2 k_{B}}{m \beta} \sum_{j=1}^{N} \int_{0}^{+\infty}\left\{\mathbb{K} e^{-\mathbb{K} \xi} \mathbb{E}_{j} e^{-\mathbb{K} \xi} \mathbb{K}\right\}_{i i} d \xi \delta T_{j} .
\end{aligned}
$$

We remark that the fluxes are given by a linear combination of the temperature jumps $\delta T_{j}$. To write these expressions in the standard way used in non-equilibrium thermodynamics, we define the thermodynamic forces $\mathscr{X}_{i}=\frac{\delta T_{i}}{T^{2}}$ and we directly identify the phenomenological coefficients

$$
\begin{aligned}
\mathscr{L}_{i j} & =-\frac{k_{B} T^{2}}{m \beta}\left\{\mathbb{K} \Delta_{j}^{s s} \mathbb{K}\right\}_{i i} \\
& =\frac{2 k_{B} T^{2}}{m \beta} \int_{0}^{+\infty}\left\{\mathbb{K} e^{-\mathbb{K} \xi} \mathbb{E}_{j} e^{-\mathbb{K} \xi} \mathbb{K}\right\}_{i i} d \xi,
\end{aligned}
$$

describing the linear relationships $\mathscr{J}_{i}=\sum_{j=1}^{N} \mathscr{L}_{i j} \mathscr{X}_{j}$. For our harmonic system, the linearity between fluxes and forces is retrieved without any additional assumptions. We can now prove the symmetry of $\mathscr{L}_{i j}$ by means of two distinct methods.

The first demonstration starts with the simple observation that one element $a_{i i}$ on the diagonal of a matrix $\mathbb{A}$ can be written as $a_{i i}=\operatorname{tr}\left\{\mathbb{E}_{i} \mathbb{A}\right\}$. Hence, the phenomenological coefficients can be rewritten as

$$
\mathscr{L}_{i j}=-\frac{k_{B} T^{2}}{m \beta} \operatorname{tr}\left\{\mathbb{E}_{i} \mathbb{K} \Delta_{j}^{s s} \mathbb{K}\right\}
$$

where we considered the first line of Eq.(77). Now, the matrix $\mathbb{E}_{i}$ can be written as $-\frac{1}{2}\left[\mathbb{K} \Delta_{i}^{s s}+\triangle_{i}^{s s} \mathbb{K}\right]$ by using Eq.(73), thus obtaining

$$
\mathscr{L}_{i j}=\frac{k_{B} T^{2}}{2 m \beta} \operatorname{tr}\left\{\left[\mathbb{K} \Delta_{i}^{s s}+\mathbb{\Delta}_{i}^{s s} \mathbb{K}\right] \mathbb{K} \Delta_{j}^{s s} \mathbb{K}\right\}
$$

Further, the last term $\Delta_{j}^{s s} \mathbb{K}$ can be substituted by $-\mathbb{K} \Delta_{j}^{s s}-2 \mathbb{E}_{j}$, exploiting again Eq.(73). We elaborate the expression of $\mathscr{L}_{i j}$ as follows

$$
\begin{aligned}
& \mathscr{L}_{i j}=-\frac{k_{B} T^{2}}{2 m \beta} \operatorname{tr}\left\{\left[\mathbb{K} \Delta_{i}^{s s}+\triangle_{i}^{s s} \mathbb{K}\right] \mathbb{K}\left[\mathbb{K} \Delta_{j}^{s s}+2 \mathbb{E}_{j}\right]\right\} \\
& =-\frac{k_{B} T^{2}}{2 m \beta} \operatorname{tr}\left\{\mathbb{K} \Delta_{i}^{s s} \mathbb{K}^{2} \Delta_{j}^{s s}+\Delta_{i}^{s s} \mathbb{K}^{3} \Delta_{j}^{s s}\right. \\
& \left.+2 \mathbb{K} \Delta_{i}^{s s} \mathbb{K E}_{j}+2 \triangle_{i}^{s s} \mathbb{K}^{2} \mathbb{E}_{j}\right\} \\
& =-\frac{k_{B} T^{2}}{2 m \beta} \operatorname{tr}\left\{\triangle_{i}^{s s} \mathbb{K}^{2} \triangle_{j}^{s s} \mathbb{K}+\triangle_{i}^{s s} \mathbb{K}^{3} \triangle_{j}^{s s}\right. \\
& \left.+2 \mathbb{E}_{j} \mathbb{K} \Delta_{i}^{s s} \mathbb{K}+2 \Delta_{i}^{s s} \mathbb{K}^{2} \mathbb{E}_{j}\right\} \\
& =-\frac{k_{B} T^{2}}{m \beta} \operatorname{tr}\left\{\Delta_{i}^{s s} \mathbb{K}^{2}\left[\frac{1}{2} \triangle_{j}^{s s} \mathbb{K}+\frac{1}{2} \mathbb{K} \Delta_{j}^{s s}+\mathbb{E}_{j}\right]\right. \\
& \left.+\mathbb{E}_{j} \mathbb{K} \mathbb{K} \Delta_{i}^{s s} \mathbb{K}\right\} \\
& =-\frac{k_{B} T^{2}}{m \beta} \operatorname{tr}\left\{\mathbb{E}_{j} \mathbb{K} \Delta_{i}^{s s} \mathbb{K}\right\}=\mathscr{L}_{j i},
\end{aligned}
$$

where we used the cyclic property of the trace stating that the trace of a product is invariant under cyclic permutations of the arguments, and Eq.(73) several times. This demonstration is simply based on Eq.(73) and does not require its explicit solution given in Eq.(75).

In contrast, the second demonstration is based on Eq.(75) and is useful since provides an explicit form of the phenomenological coefficients that is manifestly symmetric. The second line in Eq.(77) can be rewritten as

$$
\mathscr{L}_{i j}=\frac{2 k_{B} T^{2}}{m \beta} \int_{0}^{+\infty}\left\{\mathbb{K} e^{-\mathbb{K} \xi}\right\}_{i n}\left\{\mathbb{E}_{j}\right\}_{n m}\left\{e^{-\mathbb{K} \xi} \mathbb{K}\right\}_{m i} d \xi,
$$

where we introduced the implicit sums over $n$ and $m$. The definition of $\mathbb{E}_{j}$ leads to

$$
\begin{aligned}
\mathscr{L}_{i j} & =\frac{2 k_{B} T^{2}}{m \beta} \int_{0}^{+\infty}\left\{\mathbb{K} e^{-\mathbb{K} \xi}\right\}_{i n} \delta_{j n} \delta_{j m}\left\{e^{-\mathbb{K} \xi} \mathbb{K}\right\}_{m i} d \xi \\
& =\frac{2 k_{B} T^{2}}{m \beta} \int_{0}^{+\infty}\left\{\mathbb{K} e^{-\mathbb{K} \xi}\right\}_{i j}\left\{e^{-\mathbb{K} \xi} \mathbb{K}\right\}_{j i} d \xi \\
& =\frac{2 k_{B} T^{2}}{m \beta} \int_{0}^{+\infty}\left\{\mathbb{K} e^{-\mathbb{K} \xi}\right\}_{i j}\left\{\mathbb{K} e^{-\mathbb{K} \xi}\right\}_{j i} d \xi,
\end{aligned}
$$

since $\mathbb{K}$ commutes with the matrix exponential $e^{-\mathbb{K} \xi}$ for any value of $\xi$. Given that the two quantities $\left\{\mathbb{K} e^{-\mathbb{K} \xi}\right\}_{i j}$ and $\left\{\mathbb{K} e^{-\mathbb{K} \xi}\right\}_{j i}$ are scalars, their product commutes and therefore the symmetry of $\mathscr{L}_{i j}$ is evident. As a matter of course, Eq.(82) provides an explicit and practical form for the phenomenological coefficients for the system under consideration.

The complete structure of the non-equilibrium thermodynamic is retrieved only if we are able to prove that the entropy production rate given by Eq.(67) can be written as a quadratic form in the thermodynamic forces, defined by the phenomenological coefficients, as $\frac{d S_{p}}{d t}=\sum_{i=1}^{N} \mathscr{J}_{i} \mathscr{X}_{i}=$ $\sum_{i=1}^{N} \sum_{j=1}^{N} \mathscr{L}_{i j} \mathscr{X}_{i} \mathscr{X}_{j}$. As before, we investigate the mathematical form of the rate of entropy production for $t=0$, i.e. at the beginning of the relaxation to the equilibrium. Hence, Eq.(67) 
can be rewritten as

$$
\left.\frac{d S_{p}}{d t}\right|_{t=0}=\frac{1}{m \beta T} \operatorname{tr}\left\{\mathbb{K} \mathbb{Z}^{s s} \mathbb{K}-2 k_{B} T \mathbb{K}+k_{B}^{2} T^{2}\left[\mathbb{Z}^{s s}\right]^{-1}\right\},
$$

where $\mathbb{Z}^{s s}$ is the value of $\mathbb{Z}$ for $t=0$, corresponding to the NESS regime. Now, as before, we can write this covariance matrix in terms of its perturbation in the form $\mathbb{Z}^{s s}=k_{B} T \mathbb{K}^{-1}+$ $k_{B} \triangle^{s s}$. Then, the initial rate of entropy production becomes

$$
\left.\frac{d S_{p}}{d t}\right|_{t=0}=\frac{k_{B}}{m \beta} \operatorname{tr}\left\{\mathbb{K} \frac{\Delta^{s s}}{T} \mathbb{K}-\mathbb{K}+\left[\mathbb{K}^{-1}+\frac{\triangle^{s s}}{T}\right]^{-1}\right\} .
$$

Up to this point, we have not applied any approximation based on the hypothesis of small thermodynamic forces. Nevertheless, the relationship between fluxes and forces was automatically linear [see for instance Eq.(76)], without applying any approximation. Now, if we look at Eq.(84), we notice that it can be quadratic in the perturbation $\Delta^{s s}$ only thanks to the inverse matrix $\left[\mathrm{K}^{-1}+\triangle^{s s} / T\right]^{-1}$. However, the calculation of this inverse matrix surely involves a set of higher order terms in the perturbation $\Delta^{s s}$. In this sense, we can say that the Onsager entropy production emerges in our development in the form of a nonlinear expression valid arbitrarily far from equilibrium. For this reason, Eq.(84) is an important achievement of this work since it goes beyond Onsager's theory by considering higher order terms. A similar result can be found in Ref.[77], where the authors considered the thermodynamic of an open bosonic Gaussian system, in a state far from equilibrium. Interestingly, recent techniques allow the experimental measurements of the entropy production rate in quantum systems such as micromechanical resonators or Bose-Einstein condensates, allowing the systematic assessment of these theories [102]. In addition to the exact nonlinear expression given in Eq.(84), it is important to show how it is possible to retrieve the classical linearized Onsager theory. It means that the nonlinear function in Eq.(84) should reduce to the usual quadratic form close to equilibrium. To prove this point, we have to calculate the inverse of a perturbed matrix, namely $\left[\mathrm{K}^{-1}+\triangle^{s s} / T\right]^{-1}$. For this purpose, we consider the simple matrix property

$$
[A+B]^{-1}=\mathbb{A}^{-1}-\mathbb{A}^{-1} \mathbb{B}[\mathbb{A}+\mathbb{B}]^{-1},
$$

which is valid if $\mathbb{A}+\mathbb{B}$ and $\mathbb{A}$ are non singular. It can be directly proved by multiplying both sides on the right by $\mathbb{A}+\mathbb{B}$. This relation can be used recursively eventually obtaining

$$
\begin{aligned}
{[\mathbb{A}+\mathbb{B}]^{-1} } & =\mathbb{A}^{-1}\left[\sum_{k=0}^{+\infty}(-1)^{k}\left(\mathrm{BA}^{-1}\right)^{k}\right] \\
& =\mathbb{A}^{-1}-\mathbb{A}^{-1} \mathbb{B} A^{-1}+\mathbb{A}^{-1} \mathbb{B A}^{-1} \mathbb{B A}^{-1}-. .
\end{aligned}
$$

As an example, in the second line of Eq.(86) we stopped the series at the second order in $\mathbb{B}$. We apply this approximation to Eq.(84) and, thanks to the identifications $\mathbb{A}=\mathbb{K}^{-1}$ and $\mathbb{B}=$ $\triangle^{s s} / T$, we get

$$
\left.\frac{d S_{p}}{d t}\right|_{t=0}=\frac{k_{B}}{m \beta T^{2}} \operatorname{tr}\left\{\mathbb{K} \Delta^{s s} \mathbb{K} \Delta^{s s} \mathbb{K}\right\}
$$

where we find only a second order term in the perturbation, as expected. Indeed, it should be noted that the first order terms in the perturbation are completely eliminated in the result. Again, by using the decomposition in Eq.(72), we have the quadratic form

$$
\begin{aligned}
\left.\frac{d S_{p}}{d t}\right|_{t=0} & =\frac{k_{B}}{m \beta T^{2}} \sum_{i=1}^{N} \sum_{j=1}^{N} \operatorname{tr}\left\{\mathbb{K} \Delta_{i}^{s s} \mathbb{K} \Delta_{j}^{s s} \mathbb{K}\right\} \delta T_{i} \delta T_{j} \\
& =\frac{k_{B} T^{2}}{m \beta} \sum_{i=1}^{N} \sum_{j=1}^{N} \operatorname{tr}\left\{\mathbb{K} \Delta_{i}^{s s} \mathbb{K} \Delta_{j}^{s s} \mathbb{K}\right\} \mathscr{X}_{i} \mathscr{X}_{j},
\end{aligned}
$$

where we introduced the thermodynamic forces $\mathscr{X}_{i}=\frac{\delta T_{i}}{T^{2}}$, as before. Now, we have obtained the rate of entropy production as a quadratic form in the forces $\mathscr{X}_{i}$, as requested by the linearized Onsager theory, but unfortunately this quadratic form is defined by a new set of coefficients $\mathscr{H}_{i j}$, which do not exhibit the same expression of the coefficients $\mathscr{L}_{i j}$. More explicitly, we obtained

$$
\left.\frac{d S_{p}}{d t}\right|_{t=0}=\sum_{i=1}^{N} \sum_{j=1}^{N} \mathscr{H}_{i j} \mathscr{X}_{i} \mathscr{X}_{j}
$$

where

$$
\mathscr{H}_{i j}=\frac{k_{B} T^{2}}{m \beta} \operatorname{tr}\left\{\mathbb{K} \Delta_{i}^{s s} \mathbb{K} \Delta_{j}^{s s} \mathbb{K}\right\}
$$

while we previously proved that

$$
\mathscr{L}_{i j}=-\frac{k_{B} T^{2}}{m \beta} \operatorname{tr}\left\{\mathbb{E}_{i} \mathbb{K} \Delta_{j}^{s s} \mathbb{K}\right\}
$$

To find a solution to this apparent difficulty, we can rewrite Eq.(89) in the form

$$
\left.\frac{d S_{p}}{d t}\right|_{t=0}=\sum_{i=1}^{N} \sum_{j=1}^{N}\left[\left(\mathscr{H}_{i j}-\mathscr{L}_{i j}\right)+\mathscr{L}_{i j}\right] \mathscr{X}_{i} \mathscr{X}_{j},
$$

where we have adopted the expedient of adding and subtracting $\mathscr{L}_{i j}$ to $\mathscr{H}_{i j}$. If we are able to prove that the coefficients $\mathscr{A}_{i j}=\mathscr{H}_{i j}-\mathscr{L}_{i j}$ are skew-symmetric, i.e. that $\mathscr{A}_{i j}=-\mathscr{A}_{j i}$, we can accept that $\mathscr{H}_{i j} \neq \mathscr{L}_{i j}$ because it remains true that $\sum_{i=1}^{N} \sum_{j=1}^{N} \mathscr{H}_{i j} \mathscr{X}_{i} \mathscr{X}_{j}=\sum_{i=1}^{N} \sum_{j=1}^{N} \mathscr{L}_{i j} \mathscr{X}_{i} \mathscr{X}_{j}$. Indeed, a quadratic form that is constructed using a skew-symmetric matrix always takes the value zero, i.e. $\sum_{i=1}^{N} \sum_{j=1}^{N} \mathscr{A}_{i j} \mathscr{X}_{i} \mathscr{X}_{j}=$ $0 \forall \mathscr{X}_{i}$ if $\mathscr{A}_{i j}=-\mathscr{A}_{j i}$. At this point, we must therefore try to prove that $\mathscr{A}_{i j}$ is skew-symmetric. We start with its definition

$$
\mathscr{A}_{i j}=\frac{k_{B} T^{2}}{m \beta} \operatorname{tr}\left\{\mathbb{K} \Delta_{i}^{s s} \mathbb{K} \Delta_{j}^{s s} \mathbb{K}+\mathbb{E}_{i} \mathbb{K} \Delta_{j}^{s s} \mathbb{K}\right\},
$$

where we used the expressions for $\mathscr{H}_{i j}$ and $\mathscr{L}_{i j}$ given in Eqs.(90) and (91). Now, in the first product we exchange the order of the two groups of factors $\mathbb{K} \Delta_{i}^{s s}$ and $\mathbb{K} \Delta_{j}^{s s} \mathbb{K}$. Then, we get

$$
\mathscr{A}_{i j}=\frac{k_{B} T^{2}}{m \beta} \operatorname{tr}\left\{\mathbb{K} \Delta_{j}^{s s} \mathbb{K} \mathbb{K} \Delta_{i}^{s s}+\mathbb{E}_{i} \mathbb{K} \Delta_{j}^{s s} \mathbb{K}\right\} .
$$


Further, in the first product the last term $\mathbb{K} \Delta_{i}^{s s}$ can be substituted by $-\triangle_{i}^{s s} \mathbb{K}-2 \mathbb{E}_{i}$, using Eq.(73). We obtain

$$
\begin{aligned}
\mathscr{A}_{i j} & =\frac{k_{B} T^{2}}{m \beta} \operatorname{tr}\left\{-\mathbb{K} \Delta_{j}^{s s} \mathbb{K} \Delta_{i}^{s s} \mathbb{K}-2 \mathbb{K} \Delta_{j}^{s s} \mathbb{K} \mathbb{E}_{i}+\mathbb{E}_{i} \mathbb{K} \Delta_{j}^{s s} \mathbb{K}\right\} \\
& =\frac{k_{B} T^{2}}{m \beta} \operatorname{tr}\left\{-\mathbb{K} \Delta_{j}^{s s} \mathbb{K} \Delta_{i}^{s s} \mathbb{K}-\mathbb{E}_{i} \mathbb{K} \Delta_{j}^{s s} \mathbb{K}\right\} .
\end{aligned}
$$

Here, we can apply the Onsager reciprocal relations, proved in Eq.(80), to the second term, eventually obtaining

$$
\mathscr{A}_{i j}=\frac{k_{B} T^{2}}{m \beta} \operatorname{tr}\left\{-\mathbb{K} \Delta_{j}^{s s} \mathbb{K} \triangle_{i}^{s s} \mathbb{K}-\mathbb{E}_{j} \mathbb{K} \triangle_{i}^{s s} \mathbb{K}\right\} .
$$

If we now draw a comparison between Eq.(93) and Eq.(96), we immediatly deduce that $\mathscr{A}_{i j}=-\mathscr{A}_{j i}$, as we wanted to demonstrate. Finally, the fact that $\mathscr{A}_{i j}$ is skew-symmetric allows us to conclude that the rate of entropy production can be written as

$$
\left.\frac{d S_{p}}{d t}\right|_{t=0}=\sum_{i=1}^{N} \sum_{j=1}^{N} \mathscr{L}_{i j} \mathscr{X}_{i} \mathscr{X}_{j}
$$

with the same phenomenological coefficient describing the relation between fluxes and forces, i.e.

$$
\mathscr{J}_{i}=\sum_{j=1}^{N} \mathscr{L}_{i j} \mathscr{X}_{j}
$$

where the Onsager reciprocal relations $\mathscr{L}_{i j}=\mathscr{L}_{j i}$ are satisfied. We finally proved that both the heat fluxes and the entropy production obtained through the formalism of the stochastic thermodynamics are perfectly consistent with the Onsager theory during the relaxation to the equilibrium. We remark that the structure of the non-equilibrium thermodynamics summed up by Eqs.(97) and (98) is only an approximation of the general nonlinear results obtained through the stochastic thermodynamics, valid for arbitrary values of the jumps $\delta T_{i}$ or, equivalently, for states far from equilibrium. More precisely, we remember that the relation between fluxes and forces is linear also for states arbitrarily far from equilibrium, while the rate of entropy production shows a nonlinear form if we consider the result of the stochastic thermodynamics without linearization. This calculation thus shows in a deeper way the structure of the Onsager theory when treating systems arbitrarily far from equilibrium. And also proves that the mathematical structure of the Onsager theory is not easily and directly deducible from the formalism of stochastic thermodynamics even in the case in which weak thermodynamic forces are assumed. Interestingly enough, we presented here the analysis of the overdamped system, but we carefully verified that all the results can be also demonstrated with the underdamped version of the same system, accepting however some more mathematical complications. We have chosen to present only the overdamped version because it contains all the essential elements, which are crucial for the understanding of the underlying physics.

\section{CONCLUSIONS}

In this work, we presented two examples through which we discussed the relation between stochastic thermodynamics and non-equilibrium thermodynamics. In both cases, we observed the approach to equilibrium in given harmonic systems and we identified the thermodynamic fluxes and forces, characteristic of the Onsager theory. While the first example considers a system with one degree of freedom, the second one deals with $N$ degrees of freedom, coupled with the most general harmonic interaction. It means that the elastic constants can be assigned to the edges of a complete graph, describing the interactions between the particles. Since the elastic constants are arbitrary, no a priori symmetry defines the system. The idea is to consider a system simple enough to be examined analytically but sufficiently complex to have no symmetries and to be described by an arbitrary number of degrees of freedom. We identified thermodynamic forces and fluxes describing the system during the relaxation to equilibrium and we proved that they are linked through a linear map, also for states arbitrarily far from equilibrium. This linear map is characterized by coefficients satisfying the Onsagar reciprocal relations, for which we provided an ad hoc demonstration. We also presented an explicit integral expression of such Onsager coefficients, which can be applied to most of linear systems. On the other hand, the entropy production is found in general as a complex nonlinear function of the thermodynamic forces, and the classical Onsager quadratic form is retrieved only for small values of the forces, consistently with the assumption of the non-equilibrium thermodynamics. We gave an explicit demonstration that this quadratic form is based on the same set of coefficients describing the linear map between forces and fluxes, as stated within the non-equilibrium thermodynamics. Since this work is oriented to explain the relationship between stochastic thermodynamics and non-equilibrium thermodynamics, the type of system considered is rather abstract but we can say that the results obtained are valid for any physical linear system, which can also be found by linearization of an arbitrarily nonlinear system around a given work point. This means that, for example, the adopted system is suitable to represent the approach to the equilibrium of a solid or a fluid, which is composed of a collection of atoms connected by linear springs in the harmonic limit and subjected to an initial heterogeneous temperature. And it is not difficult to extend this procedure to other physical systems of interest. Although our analysis is restricted to the specific case of harmonic systems, the nonlinear form of the entropy production provides an example of particular complex behavior, which emerges when a system is driven arbitrarily far from equilibrium. As mentioned above, we recall that we presented the analysis for an overdamped system but similar results were also demonstrated for the underdamped case. Further generalizations will be considered and concern the three-dimensional extension, the application of arbitrary forces and the presence of an external magnetic field. 


\section{ACKNOWLEDGMENTS}

The author would like to thank P.-M. Déjardin and the anonymous reviewers for their constructive comments on the manuscript.

[1] R. Brown, Philos. Mag. N. S. 4, 161 (1828).

[2] R. Brown, Philos. Mag. N. S. 6, 161 (1829).

[3] A. Fick, Ann. Phys. (Leipzig) 19, 371 (1855).

[4] A. Einstein, Ann. Phys. (Leipzig) 17, 549 (1905).

[5] A. Einstein, Ann. Phys. (Leipzig) 19, 371 (1906)

[6] M. von Smoluchowski, Ann. Phys. (Leipzig) 21, 756 (1906).

[7] P. Langevin, C. R. Acad. Sci. (France) 146, 530 (1908).

[8] J. B. Perrin, C. R. Acad. Sci. (France) 158, 1168 (1914).

[9] A. D. Fokker, Ann. Phys. 348, 810 (1914).

[10] M. Planck, Sitzber. Preuss. Akad. Wiss. 324 (1917).

[11] A. Kolmogoroff, Mathematische Annalen 104, 415 (1931).

[12] O. Klein, Arkiv für Matematik, Astronomi och Fysik 16, 1 (1921).

[13] H. A. Kramers, Physica 7, 284 (1940).

[14] S. Chandrasekhar, Rev. Mod. Phys. 15, 1 (1943).

[15] W. Coffey, Advances in Chemical Physics, Vol.LXIII (1985).

[16] H. Risken, The Fokker-Planck equation, (Springer Verlag, Berlin, 1989).

[17] W. T. Coffey, Yu. P. Kalmykov, and J. P. Waldron, The Langevin equation, (World Scientific, Singapore, 2004).

[18] K. Sekimoto, J. Phys. Soc. Jpn. 66, 1234 (1997).

[19] K. Sekimoto, Stochastic Energetics (Springer, Berlin 2010).

[20] U. Seifert, Phys. Rev. Lett. 95, 040602 (2005).

[21] U. Seifert, Eur. Phys. J. B 64, 423 (2008).

[22] U. Seifert, Rep. Prog. Phys. 75, 126001 (2012).

[23] C. van den Broeck, Stochastic thermodynamics, in Selforganization by Nonlinear Irreversible Processes, Proc. 3rd Int. Conf. (Kühlungsborn, GDR, 18-22 March 1985), Eds. W. Ebeling and H. Ulbricht, pp. 57-61 (Springer, Berlin, 1985).

[24] T. Tomé and M. J. De Oliveira, Stochastic Dynamics and Irreversibility (Springer, Berlin, 2015).

[25] J. Schnakenberg, Rev. Mod. Phys. 48, 571 (1976).

[26] M. Esposito and C. Van den Broeck, Phys. Rev. E 82, 011143 (2010).

[27] C. Van den Broeck and M. Esposito, Phys. Rev. E 82, 011144 (2010).

[28] T. Tomé and M. J. de Oliveira, Phys. Rev. E 82, 021120 (2010).

[29] T. Tomé and M. J. de Oliveira, Phys. Rev. Lett. 108, 020601 (2012).

[30] T. Tomé and M. J. de Oliveira, Phys. Rev. E 91, 042140 (2015).

[31] C. Jarzynski, Phys. Rev. Lett. 78, 2690 (1997).

[32] C. Jarzynski, Phys. Rev. E 56, 5018 (1997).

[33] G. Crooks, Phys. Rev. E 60, 2721 (1999).

[34] D. Collin, F. Ritort, C. Jarzynski, S. B. Smith, I. Tinoco, C. Bustamante, Nature 437, 231 (2005).

[35] C. Jarzynski, Comptes Rendus Physique 8, 495 (2007).

[36] M. Esposito, U. Harbola, S. Mukamel, Rev. Mod. Phys. 81, 1665 (2009).

[37] S. R. de Groot and P. Mazur, Non-Equilibrium Thermodynamics (North-Holland, Amsterdam, 1962).

[38] I. Prigogine, Introduction to Thermodynamics of Irreversible Processes (Interscience, New York, 1965).
[39] L. Onsager, Phys. Rev. 37, 405 (1931).

[40] L. Onsager, Phys. Rev. 38, 2265 (1931).

[41] D. G. Miller, Chem. Rev. 60, 15-37 (1960).

[42] H. B. G. Casimir, Rev. Mod. Phys. 17, 343 (1945).

[43] H. B. G. Casimir, Proc. IEEE 51, 1570 (1963).

[44] S. R. de Groot and P. Mazur, Phys. Rev. 94, 218 (1954).

[45] S. R. de Groot and P. Mazur, Phys. Rev. 94, 224 (1954).

[46] L. Peng, Y. Zhu, and L. Hong, Phys. Rev. E 97, 062123 (2018).

[47] R. Luo, G. Benenti, G. Casati, and J. Wang, Phys. Rev. Research 2, 022009(R) (2020).

[48] F. Ritort, J. Phys.: Condens. Matter 18, R531 (2006).

[49] O. K. Dudko, Quarterly Reviews of Biophysics 49, 1-14 (2015).

[50] M. Benedito, S. Giordano, Phys. Rev. E 98, 052146 (2018).

[51] K. Svoboda, C. Schmidt, B. Schnapp, S. Block, Nature 365, 721 (1993).

[52] P. Hänggi, F. Marchesoni, Rev. Mod. Phys. 81, 387 (2009).

[53] M. Caruel and L. Truskinovsky, Phys. Rev. E 93, 062407 (2016).

[54] M. Caruel and L. Truskinovsky, Rep. Prog. Phys. 81, 036602 (2018).

[55] G. Puglisi and L. Truskinovsky, Phys. Rev. E 87, 032714 (2013).

[56] P. Benetatos, A. von der Heydt, and A. Zippelius, New J. Phys. 16, 113037 (2014).

[57] G. Florio, G. Puglisi, and S. Giordano, Phys. Rev. Research 2, 033227 (2020).

[58] P. G. Steeneken, K. Le Phan, M. J. Goossens, G. E. J.Koops, G. J. A. M. Brom, C. van der Avoort, and J. T. M. van Beek, Nat.Phys. 7, 354 (2011).

[59] V. Blickle and C. Bechinger, Nat. Phys. 8, 143 (2011).

[60] J. V. Koski, V. F. Maisi, J. P. Pekola, and D. V. Averin, Proc. Natl. Acad. Sci. U.S.A. 111, 13786 (2014).

[61] P. M. Déjardin, D. S. F. Crothers, W. T. Coffey, and D. J. McCarthy, Phys. Rev. E 63, 021102 (2001).

[62] W. T. Coffey and Y. P. Kalmykov, J. Appl. Phys. 112, 121301 (2012).

[63] S. Giordano, Y. Dusch, N. Tiercelin, P. Pernod, V. Preobrazhensky, Eur. Phys. J. B 86, 249 (2013).

[64] S. Giordano, Y. Dusch, N. Tiercelin, P. Pernod, V. Preobrazhensky, J. Phys. D: Appl. Phys. 46, 325002 (2013).

[65] S. Bandopadhyay, D. Chaudhuri, and A. M. Jayannavar, J. Stat. Mech. 2015, P1100 (2015).

[66] A. Dhar, Adv. Phys. 57, 457 (2008).

[67] S. Lepri, Thermal Transport in Low Dimensions (Springer International Publishing, Switzerland, 2016).

[68] P. L. Palla, G. Patera, F. Cleri and S. Giordano, Phys. Scr. 95, 075703 (2020).

[69] G. E. Uhlenbeck, and L. S. Ornstein, Phys. Rev. 36, 823 (1930).

[70] M. C. Wang, and G. E. Uhlenbeck, Rev. Mod. Phys. 17, 323 (1945).

[71] M. Lax, Rev. Mod. Phys. 32, 25 (1960). 
[72] C. Godrèche and J.-M. Luck, J. Phys. A: Math. Theor. 52, 035002 (2019).

[73] G. T. Landi, T. Tomé and M. J. de Oliveira, J. Phys. A: Math. Theor. 46, 395001 (2013).

[74] U. Basu, C. Maes, and K. Netočný, New J. Phys. 17, 115006 (2015).

[75] J. A. McLennan, Phys. Rev. 115, 1405 (1959).

[76] W. T. B. Malouf, J. P. Santos, L. A. Correa, M. Paternostro, and G. T. Landi, Phys. Rev. A 99, 052104 (2019).

[77] D. S. P. Salazar and G. T. Landi, Phys. Rev. Research 2, 033090 (2020).

[78] R. García-García, Phys. Rev. E 86, 031117 (2012).

[79] A. Kutvonen, T. Ala-Nissila, and J. Pekola, Phys. Rev. E 92, 012107 (2015).

[80] J. P. S. Bizarro, Phys. Rev. E 78, 021137 (2008).

[81] J. P. S. Bizarro, J. Appl. Phys. 108, 054907 (2010).

[82] J. P. S. Bizarro, Ann. Phys. (N. Y.) 363, 457 (2015).

[83] P. Jung, Phys. Rep. 234, 175 (1993).

[84] Y. Izumida and K. Okuda, Phys. Rev. E 80, 021121 (2009).

[85] Y. Izumida and K. Okuda, Eur. Phys. J. B 77, 499 (2010).

[86] K. Brandner, K. Saito, and U. Seifert, Phys. Rev. X 5, 031019 (2015).

[87] K. Proesmans and C. Van den Broeck, Phys. Rev. Lett. 115, 090601 (2015).

[88] K. Proesmans, B. Cleuren, and C. Van den Broeck, J. Stat. Mech. (2016) 023202.
[89] A. Rosas, C. Van den Broeck, K. Lindenberg, Phys. Rev. E 94, 052129 (2016)

[90] A. Rosas, C. Van den Broeck, K. Lindenberg, Phys. Rev. E 97, 062103 (2018).

[91] A. L. L. Stable, C. E. Fernández Noa, W. G. C. Oropesa, and C. E. Fiore, Phys. Rev. Research 2, 043016 (2020).

[92] S. Ray and A. C. Barato, Phys. Rev. E 96, 052120 (2017).

[93] A. C. Barato, R. Chetrite, A. Faggionato, and D. Gabrielli, New J. Phys. 20, 103023 (2018).

[94] C. E. Fiore and M. J. de Oliveira, Phys. Rev. E 99, 052131 (2019).

[95] B. A. N. Akasaki, M. J. de Oliveira, and C. E. Fiore, Phys. Rev. E 101, 012132 (2020).

[96] F. Manca, P.-M. Déjardin, S. Giordano, Annalen der Physik (Berlin) 528, 381 (2016).

[97] S. Giordano, Eur. Phys. J. B 92, 174 (2019).

[98] A. Fronczak, P. Fronczak, and J. A. Hołyst, Phys. Rev. E. 76, 061106 (2007).

[99] F. R. Gantmacher, The theory of matrices, Vol. 1 and 2 (Chelsea Publishing Company, New York, 1959).

[100] P. Lancaster and M. Tismenetsky, The theory of matrices with applications (Academic, New York, 1985).

[101] W. L. Brogan, Modern Control Theory (Prentice Hall, New Jersey, 1991).

[102] M. Brunelli, L. Fusco, R. Landig, W. Wieczorek, J. HoelscherObermaier, G. Landi, F. L. Semião, A. Ferraro, N. Kiesel, T. Donner, G. De Chiara, and M. Paternostro, Phys. Rev. Lett. 121, 160604 (2018). 